\title{
Potential benefits of anticoagulant doses of low molecular weight heparin in COVID-19: An observational retrospective study
}

\author{
Daniela Aschieri, ${ }^{1 *}$ Marco Stabile, ${ }^{2 *}$ Cristina Maestri, ${ }^{3}$ Luca Rosato, ${ }^{2}$ Michela Giovanna Coccia, ${ }^{1}$ \\ Paola Novara, ${ }^{1}$ Gianluca Lanati, ${ }^{1}$ Mariateresa Di Dio, ${ }^{1}$ Cosimo Franco,${ }^{4}$ Giuseppina Bisceglie, ${ }^{6}$ \\ Giuseppe Leddi, ${ }^{5}$ Enrico Fabrizi, ${ }^{7}$ Paola Rucci, ${ }^{8}$ Gloria Taliani ${ }^{9}$ \\ ${ }^{1}$ Cardiology Unit, Emergency Department, Castel San Giovanni Hospital, ASL of Piacenza, Castel San \\ Giovanni; ' 2 Plastic Surgery Unit, Surgery Department, Castel San Giovanni Hospital, ASL of Piacenza, \\ Castel San Giovanni; ${ }^{3}$ Emergency Care Unit, Castel San Giovanni Hospital, ASL of Piacenza, Castel San \\ Giovanni; ${ }^{4}$ Respiratory Intensive Care Unit, Castel San Giovanni Hospital, ASL of Piacenza, Castel San \\ Giovanni; ${ }^{5}$ Orthopedic Unit, Castel San Giovanni Hospital, ASL of Piacenza, Castel San Giovanni; ${ }^{6}$ General \\ Medicine Unit, Castel San Giovanni Hospital, ASL of Piacenza, Castel San Giovanni; ${ }^{7}$ DISES and DSS, \\ Università Cattolica del S. Cuore, Piacenza; ${ }^{8}$ Department of Biomedical and Neuromotor Sciences, Alma \\ Mater Studiorum University, Bologna; ${ }^{9}$ Department of Translational and Precision Medicine, Sapienza \\ University, Rome, Italy
}

\begin{abstract}
Correspondence: Marco Stabile, Plastic Surgery Unit, Surgery Department, Castel San Giovanni Hospital, ASL of Piacenza, Viale 2 Giugno 1, 29015 Castel San Giovanni (Piacenza), Italy.

Tel.: +39 3397974402

E-mail:marcostabile@gmail.com

Key words: Pneumonia; COVID-19; thrombosis; heparin; mortality.

Acknowledgements: Special thanks to the members of Castel San Giovanni COVID-Speciality Hospital: Francesco Andrani, Angelo Benedetti, Mara Bozzarelli, Carlo Cagnoni, Paolo Cambrini, Esther Centenara, Sara Chiesa, Mauro Codeluppi, Patrizia Colazzo, Donatella Covini, Paolo Magri, Angelo Mangia, Demostene Marifoglou, Massimiliano Mazzilli, Samantha Mazzocchi, Monica Pellegrini, Iacopo Pellegrino, Annamaria Pieri, Daria Sacchini, Anna Chiara Vermi, Corrado Tosca, Lucia Torretta, Gioacchino Valenti, Ercole Zanotti.

Contributions*: DA and MS contributed equally.

Conflicts of interest: The authors declare no conflict of interest.
\end{abstract}

Availability of data and material: Data are available upon request from the first author.

Ethics approval and consent to participate: The Healthcare Directorate of Piacenza Local Health Authority approved the therapeutic protocol. All methods involving patients, data and information were carried out in accordance with relevant guidelines and regulations. The Healthcare Directorate of Piacenza Local Health Authority granted a waiver of informed consent.

Informed consent: No consent for publication was requested, because data are presented in aggregate form and do not include sensitive data.

Received for publication: 31 March 2021.

Revision received: 4 August 2021.

Accepted for publication: 6 August 2021.

This work is licensed under a Creative Commons Attribution 4.0 License (by-nc 4.0).

${ }^{\circ}$ Copyright: the Author(s), 2021

Licensee PAGEPress, Italy

Emergency Care Journal 2021; 17:9795

doi:10.4081/ecj.2021.9795

\begin{abstract}
Hypercoagulability is a common complication of the systemic inflammation related to coronavirus disease 2019 creating debate within the critical care community on the therapeutic utility of Low Molecular Weight Heparin (LMWH). We collected data on consecutive patients with COVID-19 admitted to the Emergency Department of Castel San Giovanni Hospital, between February 29th and April 7th, 2020. Exclusion criteria were age $<18$ years, hospital stay $<7$ days, patients on dialysis and patients who had been transferred to other centers for which we could not collect data. Of the 257 patients included in the study, 49 (19.1\%) died during hospitalization. We considered a wide set of variables as independent variables (age, sex, comorbidities and in-hospital treatments). We used a multivariate logistic regression model and, being heparin the only one therapy affecting survival rate, we compared prophylactic LMWH (p-LMWH) and Therapeutic LMWH (T-LMWH) groups. Kaplan Meier curve showed a higher survival probability in the T-LMWH and the difference between the two groups was statistically significant according to the log-rank or Mantel- Haenszel test $(p<0.0001)$. In a stratified analysis by ventilation type, the subgroup of patients who benefited from therapeutic LMWH was that in non-invasive mechanical ventilation. Using a multivariate analysis and adjusting for the drugs intake, TLMWH was the only therapy impacting on survival (HR 0.293, p $<0.001)$. No fatal bleeding was observed. Therapeutic dose of LMWH in patients admitted to hospital with COVID-19 pneumonia was 70 associated with a decrease risk of intra-hospital mortality.
\end{abstract}

\section{Introduction}

Coronavirus disease 2019 (COVID-19) is a systemic infection characterized by high risk of death; it was first reported in Wuhan, the capital city of Hubei, in December 2019. The first case in Italy was reported on February 21, 2020 and, a few days later, Castel San Giovanni Hospital was entirely dedicated to the treatment of COVID-19 patients. No specific pharmacological protocols with proved efficacy were available for the treatment of COVID-19 pneumonia patients. ${ }^{1}$ Pneumonia with respiratory failure is the main clinical feature of COVID-19 and it is often associated with 
systemic inflammation that induces diffuse endothelial injury and hypercoagulability state. ${ }^{2-5}$ The relationship between viral infections and thrombosis is recognized, but it seems to be predominant in SARS-CoV2 infection, leading to life-threatening thrombotic complications such as venous and arterial thrombosis, pulmonary embolism and microvascular thrombi. ${ }^{6-8}$

Low Molecular Weight Heparin (LMWH) is recommended in COVID-19 patients by some expert consensus, but its efficacy and safety at therapeutic doses has not been described yet.9-11

We assumed that LMWH at therapeutic doses could exert a beneficial effect on the clinical course of COVID-19 thanks to its anticoagulant, anti-inflammatory and in vitro antiviral properties. ${ }^{12-16}$

The aim of this observational study was to estimate the effect of anticoagulation on the in-hospital mortality of COVID-19.

\section{Materials and Methods}

We conducted an observational study collecting data of 351 adult patients consecutively admitted to Castel San Giovanni Hospital from February 29th to April 7th, 2020 with confirmed COVID-19 pneumonia. 257/351 patients were included in this study. The COVID-19 diagnosis was made according to the World Health Organization interim guidelines and was confirmed by RNA detection of SARS-CoV-2 from oropharyngeal and nasopharyngeal swab sample. ${ }^{6}$ High Resolution Computed Tomography (HRCT) scan diagnosis of pulmonary pneumonia was performed in all 100 patients.

Exclusion criteria were age $<18$ years, hospital stay $<7$ days, patient on dialysis and patients who had been transferred to other centers for further treatment for which we could not collect data. We collected data on age, sex, comorbidity (arterial hypertension, diabetes mellitus, cardiovascular disease, chronic or paroxysmal atrial fibrillation, COPD, chronic kidney disease, history of cancer, obesity, smoke, dyslipidemia) and in hospital therapy (corticosteroid, azithromycin, hydroxychloroquine, tocilizumab, anti-viral therapy and oxygen support).

All patients included received LMWH within 24 hours of admission until March $16^{\text {th }}$.

LMWH was administered at a prophylactic dose of $4000 \mathrm{UI}$ once per day [prophylaxis of Deep Vein Thrombosis (DVT)/Pulmonary Embolism (PE) in bed ridden patients] for at least 7 days. ${ }^{17,18}$ After a collegial discussion between the main directors of the COVID Center operating units, based on the evidence of the anti-inflammatory effect of heparin and given the initial evidence of important states of hypercoagulability, it was decided to contact the local authority (Healthcare Directorate of Piacenza) and ask for consent to use of Therapeutic, anticoagulant dose of LMWH (T-LMWH) in all COVID patients within 24 from the admission. Obviously the T-LMWH was adjusted for patient's weight and Estimated Glomerular Filtration Rate (eGFR). ${ }^{17,18}$ Patients with HAS-BLED risk $>3$ were treated with $\mathrm{p}-\mathrm{LMWH}$. The new therapeutic protocol started on 16th March.

\section{Data Collection}

Data were extracted from electronic medical records and included age, gender, clinical characteristics, in-hospital pharmacological treatments and type of oxygenation/ventilation needed.

The comorbidities extracted were arterial hypertension, diabetes mellitus, cardiovascular diseases (defined as history of myocardial infarction, ischemic stroke, and peripheral atheromasia), atrial fibrillation, chronic obstructive pulmonary disease, chronic kidney disease (Cockroft-Gault e-GFR $<60$ $\mathrm{mL} / \mathrm{min} / 1.73 \mathrm{~m}^{2}$ ) and the presence of active or previous cancer. Bleeding events were also extracted and classified according to the International Society of Thrombosis and Hemostasis (ISTH) definition of bleeding. ${ }^{19}$

\section{Statistical analysis}

We considered as independent variables age, sex and a set of variables described in comorbidities and in-hospital treatments listed in Table 1. To explore the impact of all these variables on mortality, we first performed univariate analysis estimating the odds ratios of mortality, for each variable, along with their confidence intervals. Then, a multivariate logistic regression model was used including significant variables identified in univariate analyses.

Being LMWH the only one therapy affecting survival rate, we subdivided the population in two groups, $\mathrm{P}$ and T-LMWH, and then compared the two groups of patients using two sample tests: $\mathrm{z}$ test for dichotomous variables, chi-square test for categorical variables, while Wilicoxon Mann-Whitney was used for the comparison of distributions of numerical variables. For LMWH dose we also used a z-test for comparing proportions, along with analysis of its impact on patients survival over the observation period. To this latter aim, a graphical representation of the Kaplan-Meier curves, separated for the two groups, was done and compared using long-rank or Mantel-Haenszel test. The z test (with continuity correction) was also applied to the analysis of samples stratified according to the ventilation level. Moreover a cox regression model was used to adjusting results for drug intake, focusing on those that statistically differ between the two groups.

All statistical computation were carried out using the software $\mathrm{R}$ version 3.5.1

\section{Results}

Between February 29th and April 7th, 2020, 351 patients were admitted to Castel San Giovanni Hospital. Ninety-four patients were excluded from the analysis for the following reasons: within 7 days 30 of them were discharged, 43 died and 17 were transferred to other care units and we had no data to collect, 3 were not COVID-19 confirmed patients and 1 patient was undergoing hemodialysis and excluded. One hundred thirty-one (51\%) received prophylactic LMWH and 126 patients (49\%) received LMWH (each clinician adjusted the therapeutic dose of heparin based on each patient's weight and eGFR).

Two hundred-thirty-two patients $(90.3 \%)$ received empiric antibiotic treatment (azithromycin $500 \mathrm{mg}$ /day for at least 5 days), 237 patients $(92.2 \%)$ received hydroxychloroquine (200 $\mathrm{mg}$ twice a day for at least 5 days), 146 patients $(55.7 \%)$ received corticosteroids (methylprednisolone $1 \mathrm{mg} / \mathrm{Kg}$ twice a day i.v. or dexamethasone $40 \mathrm{mg}$ i.v. once a day for 3 days followed by decreasing dosage), 236 patients $(91.8 \%)$ received anti-retroviral therapy (darunavir/cobicistat $800+150 \mathrm{mg}, 1$ tablet for 7 days and/or lopinavir/ritonavir $200+50 \mathrm{mg}, 1$ tablet twice a day for 7 days). Twenty-five

patients $(9.5 \%)$ were eligible for treatment with tocilizumab $(8$ $\mathrm{mg} / \mathrm{kg}$ i.v. in a single or double dose). D-dimer levels were collected in 93 patients, and the mean peak level was $5241 \mu \mathrm{g} / \mathrm{mL}$; the median level was $1489 \mu \mathrm{g} / \mathrm{mL}$. All patients required oxygen therapy: 135 patients $(52,5 \%)$ needed nasal cannulas with oxygen flow $\leq 6 \mathrm{l} / \mathrm{min}$ or non-rebreather mask with oxygen flow from 10 to 15 $1 /$ min (Vent 1$)$; 67 patients $(26.1 \%$ ) required non-invasive mechan- 
ical ventilation with Helmet C-PAP (Vent 2) and 55 patients $(21.4 \%)$ required invasive mechanical ventilation (Vent 3 ). Patients treated with prophylactic and therapeutic LMWH were compared for demographic and clinical characteristics (Table 1).

\section{In-hospital mortality of patients treated with therapeu- tic LMWH vs. prophylactic LMWH}

During hospitalization, 49 patients included died (49/257, $19.1 \%$ ). Univariate logistic regression with odd ratios and $95 \%$ confidence intervals first documented the impact of heparin, older age and Invasive Ventilation on mortality (Table 2). Multivariate logistic regression analysis documented that older age and Invasive mechanical Ventilation were associated with an increased risk of death and reinforced the statistical significance of the impact of LMWH on survival (Table 3).

Moreover, comparing $\mathrm{P}$ and T-LMWH groups and the impact on patients survival over the observational period, Kaplan Meier curve showed an higher survival probability in the T-LMWH and the difference between the two groups was statistically significant according to the log-rank or Mantel-Haenszel test $(p<0.0001$; Figure 1). Using a multivariate regression model and adjusting the date for the two drugs that significantly varied between the two groups, T-LMWH was the only drug impacting on survival (Table 4). In a stratified analysis by ventilation type, the subgroup of patients 187 who benefited from T- LMWH was that in non-invasive mechanical ventilation (Table 5).

\section{Safety of therapeutic LMWH}

Two major and one minor bleeding episodes were recorded in patients treated with therapeutic LMWH dosage (3/126; $2.4 \%)$ : two patients presented psoas muscle hematoma that required transfusion of two units of packed red blood cells, and one patient gluteus muscle hematoma that recovered spontaneously. No patient needed invasive treatment.

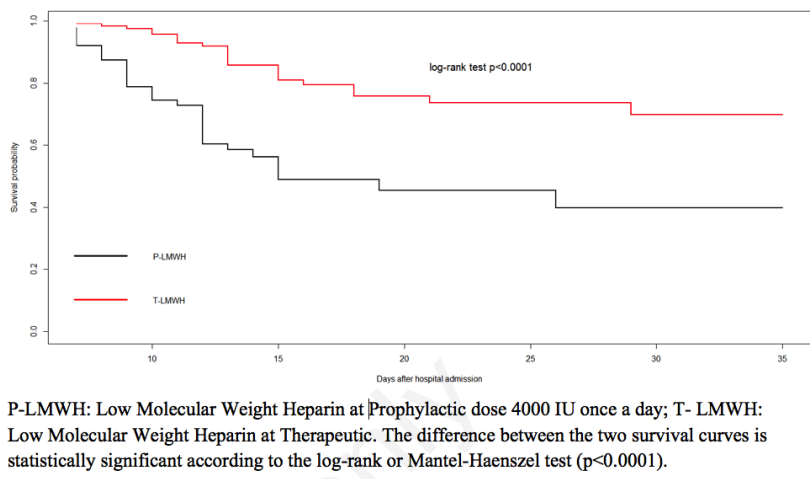

Figure 1. Kaplan-Meier survival curves of patients treated with prophylactic (P-LMWH) or therapeutic (T-LMWH) doses of Low Molecular Weight Heparin.

Table 1. Demographic, clinical characteristics and treatment of the study population and comparisons between patients treated with p- LMWH (N=131) and t- LMWH (N=126).

\begin{tabular}{|c|c|c|c|c|}
\hline Variables & $\begin{array}{c}\text { Overall sample } \\
\text { Mean } \pm \text { SD or } N(\%)\end{array}$ & $\begin{array}{c}\text { P-LMWH } \\
\text { Mean } \pm \text { SD or N }(\%)\end{array}$ & $\begin{array}{c}\text { T-LMWH } \\
\text { Mean } \pm \text { SD or N }(\%)\end{array}$ & p \\
\hline Age (years) & $69.3+10.7$ & $69.4(11.9)$ & $69.2(9.5)$ & $0.836^{\circ}$ \\
\hline $\begin{array}{l}\text { Gender } \\
\quad \text { Male } \\
\text { Female } \\
\end{array}$ & $\begin{array}{l}174(67.7) \\
83(32.3)\end{array}$ & $\begin{array}{l}91(69.5) \\
40(30.5)\end{array}$ & $\begin{array}{l}83(65.9) \\
43(34.1)\end{array}$ & $0.583^{\wedge}$ \\
\hline Arterial hypertension & $162(63.0)$ & $83(63.4)$ & $79(62.7)$ & $0.913^{\wedge}$ \\
\hline Diabetes mellitus type 2 & $46(17.9)$ & $25(19.1)$ & $21(16.7)$ & $0.613^{\wedge}$ \\
\hline Cardiovascular disease & $44(17.1)$ & $28(21.4)$ & $16(12.7)$ & $0.065^{\wedge}$ \\
\hline Dyslipidaemia & $37(14.4)$ & $19(15.0)$ & $18(14.3)$ & $0.879^{\wedge}$ \\
\hline 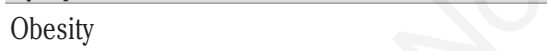 & $25(9.7)$ & $12(9.2)$ & $13(10.3)$ & $0.754^{\wedge}$ \\
\hline Smoke & $21(8.2)$ & $12(9.4)$ & $9(7.1)$ & $0.518^{\wedge}$ \\
\hline Chronic or paroxysmal atrial fibrillation & $33(12.8)$ & $17(13.0)$ & $16(12.8)$ & $0.966^{\wedge}$ \\
\hline Chronic obstructive pulmonary disease & $22(8.6)$ & $13(9.9)$ & $9(7.1)$ & $0.426^{\wedge}$ \\
\hline Chronic kidney disease & $22(8.6)$ & $14(10.8)$ & $8(6.3)$ & $0.207^{\wedge}$ \\
\hline History of cancer & $18(7.0)$ & $11(8.4)$ & $7(5.6)$ & $0.372^{\wedge}$ \\
\hline \multicolumn{5}{|l|}{ IN-HOSPITAL TREATIMENT } \\
\hline Azithromycin & $232(90.3)$ & $108(82.4)$ & $114(90.5)$ & $0.061^{\wedge}$ \\
\hline Hydroxychloroquine & $237(92.2)$ & $114(87.0)$ & $123(97.6)$ & $0.002^{\wedge}$ \\
\hline Corticosteroid & $146(56.8)$ & $44(33.6)$ & $102(81.0)$ & $<0.001^{\wedge}$ \\
\hline $\begin{array}{l}\text { Anti-viral therapy } \\
\text { Lopinavir/ritonavir and/or Darunavir/cobicistat } \\
\text { Tocilizumab }\end{array}$ & $\begin{array}{c}236(91.8) \\
25(9.7)\end{array}$ & $\begin{array}{l}118(90.1) \\
2(1.5)\end{array}$ & $\begin{array}{l}119(94.4) \\
23(18.3)\end{array}$ & $\begin{array}{l}0.191^{\wedge} \\
<0.001^{\wedge}\end{array}$ \\
\hline $\begin{array}{l}\text { Oxygen support } \\
\text { Vent } 1 \\
\text { Vent } 2 \\
\text { Vent } 3\end{array}$ & $\begin{array}{l}135(52.5) \\
67(26.1) \\
55(21.4)\end{array}$ & $\begin{array}{l}78(59.5) \\
28(21.4) \\
25(19.1)\end{array}$ & $\begin{array}{l}57(42.5) \\
39(31.0) \\
30(23.8)\end{array}$ & $0.066^{\wedge}$ \\
\hline
\end{tabular}

P-LMWH: Prophylactic Low Molecular Weight Heparin dose 4000 UI once a day; T- LMWH: Therapeutic Low Molecular Weight Heparin dose adjusted for weight and/or eGFR of each patient. Vent 1: nasal cannula with oxygen flow $<6 \mathrm{~L} / \mathrm{min}$ or non-rebreather mask with oxygen flow from 10 to $15 \mathrm{~L} / \mathrm{min}$; Vent 2 : non-invasive mechanical ventilation (Helmet C-PAP); Vent 3 : invasive mechanical ventilation. ${ }^{\wedge}$ Chi-square test. ${ }^{\circ}$-test. 


\section{Discussion}

During this "war" against the rapidly spread of COVID-19, we had not data-driven therapeutic strategies. Castel San Giovanni Hospital became the first COVID Centre in Italy and we had only few preclinical evidence to guide our treatment strategies.

As yet, there are no exhaustive data on the risk factor associated with COVID-19, due to extremely different clinical settings and insufficient follow-up.

A meta-analysis of eight studies including 46248 patients with laboratory confirmed COVID-19 indicated that those with the most severe disease were more likely to have hypertension, respiratory disease and cardiovascular disease. In other studies, obesity and smoking were associated with increased risks. ${ }^{20,21}$ Our study, reported an higher mortality risk in older patients and in patients who needed invasive ventilation support (indirectly related to the extent of lung involvement). SARS-Cov-2 infection has been shown to induce diffuse endothelial inflammation due to direct viral infection of the endothelial cells in different organs of the human body. ${ }^{22}$ The endothelial damage is the main determinant of micro vascular dysfunction that led to a vasoconstriction and a subsequent organ ischemia, inflammation, tissue edema and a procoagulant state. ${ }^{23}$

Early application of anticoagulant therapy in COVID-19 was suggesting first in China for improving outcome. Beyond anticoagulation, there may be alternative beneficial mechanisms of action for heparin in patients with COVID-19 including direct SARS-CoV-2 antiviral activity of unfractionated heparin therapy. Its effect could be partially due to this theoretical antiviral activity,

Table 2. Univariate regression analysis - odd ratios and $95 \%$ confidence interval for mortality

\begin{tabular}{lccc} 
& Odds ratio & \multicolumn{2}{c}{$95 \%$ confidence interval } \\
$(97.5 \%)$ & $(2.5 \%)$ & 0.91 \\
Heparin & & 0.29 & 2.27 \\
Sex & 0.52 & 0.66 & 1.10 \\
\hline Age (y) & 1.21 & 1.03 & 4.67 \\
Vent (level 2) & 1.06 & 1.14 & 8.61 \\
\hline Vent (level 3) & 2.31 & 2.08 & 3.55 \\
Cardiovascular disease & 4.20 & 0.89 & 4.13 \\
\hline Hypertension & 1.80 & 1.16 & 3.62 \\
Chronic kidney disease & 2.14 & 0.53 & 6.78 \\
\hline History of tumors & 1.45 & 0.93 & 4.39 \\
Chronic Obstructive Pulmonary Disease (COPD) & 2.55 & 0.68 & 1.43 \\
\hline Diabetes mellitus II & 1.78 & 0.29 & 2.80 \\
Corticosteroid & 0.67 & 0.87 & 4.98 \\
Antiviral_T & 1.55 & 0.49 & 1.86 \\
Tocilizumab & 1.38 & 0.23 & \\
\hline
\end{tabular}

Heparin (including therapeutic and prophylactic low molecular weight heparin). Vent 1: nasal cannula with oxygen flow < 6L/min or non-rebreather mask with oxygen flow from 10 to $15 \mathrm{~L} /$ min; Vent 2: non-invasive mechanical ventilation (Helmet C-PAP); Vent 3: invasive mechanical ventilation.

Table 3. Multivariate logistic regression analysis and estimated coefficients.

\begin{tabular}{|c|c|c|c|c|c|}
\hline & Estimate & Std. Error & $\mathrm{z}$ value & $\operatorname{Pr}(>|z|)$ & p \\
\hline (Intercept) & -10.563 & 2.054 & -5.142 & 0.0000 & $* * *$ \\
\hline Heparin & -1.200 & 0.414 & -2.896 & 0.0038 & $* *$ \\
\hline Sex (male) & 0.222 & 0.392 & 0.566 & 0.5711 & \\
\hline Age & 0.108 & 0.024 & 4.455 & 0.0000 & $* * *$ \\
\hline Vent (level 2) & 1.094 & 0.454 & 2.409 & 0.0160 & $*$ \\
\hline Vent (level 3) & 2.413 & 0.517 & 4.665 & 0.0000 & $* * *$ \\
\hline Cardiovascular disease & 0.414 & 0.448 & 0.925 & 0.3552 & \\
\hline Hypertension & 0.078 & 0.399 & 0.195 & 0.8454 & \\
\hline Chronic kidney disease & 0,081 & 0.594 & 0.136 & 0.8917 & \\
\hline History of tumors & 1.082 & 0.617 & 1.755 & 0.0793 & \\
\hline Chronic lung disease & 0.773 & 0.586 & 1.318 & 0.1874 & \\
\hline Diabetes mellitus II & -0.585 & 0.470 & -1.243 & 0.2139 & \\
\hline Corticosteroid & 0.565 & 0.421 & 1.342 & 0.1797 & \\
\hline antiviral_t & 0.717 & 0.719 & 0.998 & 0.3181 & \\
\hline tocilizumab & 0.153 & 0.624 & 0.245 & 0.8065 & \\
\hline
\end{tabular}

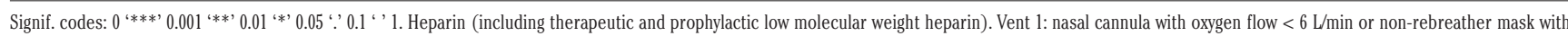
oxygen flow from 10 to $15 \mathrm{~L} / \mathrm{min}$; Vent 2: non-invasive mechanical ventilation (Helmet C-PAP); Vent 3: invasive mechanical ventilation. 
Table 4. Hazard ratio of mortality in patients treated with T- LMWH vs. P-LMWH, adjusted for concomitant drug intake (only drugs that for drugs that significantly varied between the two groups). Results of Cox regression model.

\begin{tabular}{lcccccc} 
& b & SE(b) & HR & $95.0 \%$ & & CI \\
T- LMWH vs. P-LMWH & -1.229 & 0.330 & 0.293 & 0.153 & 0.558 & 1.757 \\
Tocilizumab & -0.890 & 0.742 & 0.411 & 0.096 & 2.173 & 0.230 \\
\hline Corticosteroids & 0.155 & 0.317 & 1.167 & 0.627 & 0.626 \\
Hydroxychloroquine & 0.074 & 0.603 & 0.902 & 1.077 & 0.330 & 3.513 \\
\hline
\end{tabular}

P-LMWH: Low Molecular Weight Heparin at Prophylactic doses 4000 once a day; T- LMWH: Low Molecular Weight Heparin at Therapeutic doses.

Table 5. Stratified analysis by ventilation type.

\begin{tabular}{|c|c|c|c|c|}
\hline & & & & \\
\hline & n cases & P-LMWH & T-LMWH & $Z$ test $p$ value \\
\hline Vent 1 & 135 & 0.167 & 0.140 & 0.86 \\
\hline Vent 2 & 67 & 0.571 & 0.102 & 0.0001 \\
\hline Vent 3 & 55 & 0.48 & 0.4 & 0.747 \\
\hline
\end{tabular}

P-LMWH: Low Molecular Weight Heparin at Prophylactic doses 4000 once a day; T- LMWH: Low Molecular Weight Heparin at Therapeutic doses. Vent 1: nasal cannula with oxygen flow < 6 L/min or non-rebreather mask with oxygen flow from 10 to 15L/min; Vent 2: non-invasive mechanical ventilation (Helmet C-PAP); Vent 3: invasive mechanical ventilation.

rather than solely due to its anticoagulant properties.

Tang et al. highlighted the importance of the risk stratification (according to validated score and blood parameters) pointing out the impact of the anticoagulant therapy in particular settings; similar results were, recently, validate by a multiplatform trials. Unfortunately, due to the emergency, we didn't collect systematically laboratory parameters, therefore, we were not able to stratify the risk of the study population.

Our personal experience aims to provide a further contribution to the scientific 221 evidence in favor of the use of T-LMWH within 24 hours of hospital access in all patients with COVID-19 infection. Our observational study demonstrated that administration of T LMWH was associated with lower mortality in patients admitted with COVID-19 also adjusting data from other drug intake. Finally, we think that the therapeutic efficacy of T-LMWH is evident in the Vent-2 group where the uncontrolled inflammatory response and cytokines storm is the main pathophysiological aspect of the disease, which could culminate in extensive and irreversible lung damage requiring intubation. In this phase we can positively change the course of the disease and the history of our patients. Heparin is easy to administer and bleeding complications are unusual.

Radiological features on CT-images, in COVID-19 patients, suggest veno-artero thrombosis ${ }^{20,21}$ and also in autopsy studies has been found diffuse thrombosis of the peripheral small vessels. ${ }^{23}$ On the basis of this evidence, the role of heparin treatment was debate in the scientific community.

To date there are a lot of observational evidence and some randomized trials are ongoing but there are no solid evidences that recommends the routine use of T-LMWH due to conflicting results related to: different study population, therapeutic regimens and choice of anticoagulant therapy (sometimes preferring intermediate doses of heparin or DOAC), perhaps losing the antinflammatory and antiviral effect of heparin and increasing the risk of interactions with COVID-19 treatments; Active 4b- Anti-thrombotics for Adults Hospitalized With COVID-19 (ACTIV-4); Hero19Hamburg Edoxaban for Anticoagulation in COVID-19 Study (HERO-19); Corona virus edoxaban colchicine (convince) COVID-19 (convince) ongoing. ${ }^{24-26}$

Limitations in current study include the potential selection bias as all retrospective study. It is possible that some non-pharmacological change took place in the management of patients as the clinicians were more expert to treat this disease over the period. These results should be interpreted with caution owing to potential bias and residual confounding in this observational study with a small sample size.

\section{Conclusions}

T-LMWH improve survival rate in COVID-19 patients and seems to be safe.

Double-blinded randomized clinical trials are still needed to confirm this observational retrospective study and to identify the time, the dose and the biohumoral or clinical or instrumental data that could guide the correct strategy.

\section{References}

1. Kalil AC. Treating COVID-19-off-label drug use, compassionate use, and randomized clinical trials during pandemics. JAMA 2020;323:1897-8.

2. Chen N, Zhou M, Dong X, et al. Epidemiological and clinical characteristics of 99 cases of 2802019 novel coronavirus pneumonia in Wuhan, China: a descriptive study. Lancet 2020;395:507-13.

3. Huang C, Wang Y, Li X, et al. Clinical features of patients infected with 2019 novel coronavirus in Wuhan, China. Lancet 2020;395:497-506.

4. Tang N, Li D, Wang X, et al. Abnormal coagulation parameters are associated with poor prognosis in patients with novel coronavirus pneumonia. J Thromb Haemost 2020;18:844-7.

5. Avila J, Long B, Holladay B, Gottilieb M. Thrombotic complications of COVID-19, Am J Emerg Medicine 2021;39:213-8.

6. Klok FA, Kruip MJHA, van der Meer NJM, et al. Incidence of thrombotic complications in critically ill ICU patients with COVID-19. Thromb Res 2020;191:145-7.

7. Reed A, Howard C, Barakat M, Navi A. Simultaneous arterial 
and venous thromboembolic events associated with COVID19. A case report. Emerg Care J 2021;17:9718.

8. Helms J, Tacquard C, Severac F, et al. High risk of thrombosis in patients with severe SARS288 CoV-2 infection: a multicenter prospective cohort study. Intensive Care Med 2020;46:1089-8.

9. Thachil J, Tang N, Gando S, et al. ISTH interim guidance on recognition and management of coagulopathy in COVID-19. J Thromb Haemost 2020;18:1023-6.

10. World Health Organization. Clinical management of severe acute respiratory infection when novel coronavirus ( $\square 2019$ $\mathrm{nCoV}) \square$ infection is suspected: interim guidance, 28 January 2020. World Health Organization, 2020. Available from: https://apps.who.int/iris/handle/10665/330893

11. Tang N, Bai H, Chen X, Gong J, Li D, Sun Z. Anticoagulant treatment is associated with decreased mortality in severe coronavirus disease 2019 patients with coagulopathy. J Thromb Haemost 2020;18:1094-9.

12. Hirsh J, Warkentin TE, Shaughnessy SG, et al. Heparin and low-molecular-weight heparin: mechanisms of action, pharmacokinetics, dosing, monitoring, efficacy, and safety. Chest 2001;119:64S-94S.

13. Mousavi S, Moradi M, Khorshidahmad T, Motamedi M. AntiInflammatory Effects of Heparin and Its Derivatives: A Systematic Review. Adv Pharmacol Sci 2015;2015:507151.

14. Lin L, Lu L, Cao W, Li T. Hypothesis for potential pathogenesis of SARS-CoV-2 infection-a review of immune changes in patients with viral pneumonia. Emerg Microbes Infect 2020;9:727-32.

15. Johnson Z, Kosco-Vilbois MH, Herren S, et al. Interference with heparin binding and oligomerization creates a novel antiinflammatory strategy targeting the chemokine system. J Immunol 2004;173:5776-85.

16. Thachil J. The versatile heparin in COVID-307 19. J Thromb Haemost 2020;18:1020-2.

17. Venclauskas L, Llau JV, Jenny JY, Kjaersgaard-Andersen P.
ESA VTE Guidelines on perioperative venous thromboembolism prophylaxis: day surgery and fast track surgery. Eur J Anaesthesiol 2018;35:134-8.

18. Schünemann HJ, Cushman M, Burnett AE, et al. American Society of Hematology 2018 guidelines for management of venous thromboembolism: prophylaxis for hospitalized and nonhospitalized medical patients. Blood Adv 2018;2:3198225.

19. Kaatz S, Ahmad D, Spyropoulos AC, Schulman S; Subcommittee on Control of Anticoagulation. Definition of clinically relevant non-major bleeding in studies of anticoagulants in atrial fibrillation and venous thromboembolic disease in non-surgical patients: communication from the SSC of the ISTH. J Thromb Haemost. 2015;13:2119-26.

20. Yang J, Zheng Y, Gou X, et al. Prevalence of comorbidities and its effects in patients infected with SARS-CoV-2: a systematic review and meta-analysis. Int J Infect Dis 2020;94:91-5.

21. Livingston E, Bucher K. Coronavirus Disease 2019 (COVID19) in Italy. JAMA 2020;323:1335.

22. Bonetti PO, Lerman LO, Lerman A. Endothelial dysfunction: a marker of atherosclerotic risk. Arterioscler Thromb Vasc Biol 2003;23:168-75.

23. Wichmann D, Sperhake J, et al. Autopsy findings and venous thromboembolism in patients with COVID-19: A prospective cohort study. Ann Intern Med 2020;173:268-77.

24. Helms, C. Tacquard, F. Severac, I. et al. High risk of thrombosis in patients with severe SARS-CoV-2 infection: a multicenter prospective cohort study. Intensive Care Med 2020;46:1089-98.

25. Schutgens RE. DOAC in COVID-19: Yes or No? Hemasphere 2020;5:e526.

26. Talasaz AH, Sadeghipour P, Kakavand H. Recent randomized trials of antithrombotic therapy for patients with COVID-19: JACC state-of-the-art review. J Am Coll Cardiol 2021;77:1903-21. 